EPJ manuscript No.

(will be inserted by the editor)

\title{
Transition from fractal to non-fractal scalings in growing scale-free networks
}

\author{
Zhongzhi Zhang ${ }^{1,2}$ a, Shuigeng Zhou ${ }^{1,2}$ b, Lichao Chen ${ }^{1,2}$, and Jihong Guan ${ }^{3}$ \\ 1 Department of Computer Science and Engineering, Fudan University, Shanghai 200433, China \\ 2 Shanghai Key Lab of Intelligent Information Processing, Fudan University, Shanghai 200433, China \\ 3 Department of Computer Science and Technology, Tongji University, 4800 Cao'an Road, Shanghai 201804, China \\ Received: date / Revised version: date
}

\begin{abstract}
Real networks can be classified into two categories: fractal networks and non-fractal networks. Here we introduce a unifying model for the two types of networks. Our model network is governed by a parameter $q$. We obtain the topological properties of the network including the degree distribution, average path length, diameter, fractal dimensions, and betweenness centrality distribution, which are controlled by parameter $q$. Interestingly, we show that by adjusting $q$, the networks undergo a transition from fractal to non-fractal scalings, and exhibit a crossover from 'large' to small worlds at the same time. Our research may shed some light on understanding the evolution and relationships of fractal and non-fractal networks.
\end{abstract}

PACS. 89.75.Hc Networks and genealogical trees $-47.53 .+\mathrm{n}$ Fractals - 05.70.Fh Phase transitions: general studies

\section{Introduction}

The past ten years have witnessed a considerable interest in characterizing and understanding the topological properties of networked systems [1,2, 3, 4, 5]. It has been established that small-world property [6] and scale-free behavior 7] are the two most fundamental concepts constituting our basic understanding of the organization of many natural and social systems. A serial of recent research indicate that these two features often go along, and have important consequences on almost every aspect on dynamic processes taking place on networks 3,4 . The small-world characteristic means that the node number of network (order) increases exponentially with the average path length (APL), and thus leads to the general understanding that complex scale-free small-world networks are not invariant or topologically fractal, since fractal networks implies that there is a power-law relation between the network order and its APL.

More recently, by using a renormalization procedure based on the box-counting method, Song, Havlin and Makse discovered that some real-life networks exhibit fractal scaling 8,9 . The fractal topology can be characterized via two relevant exponents: fractal dimension $d_{B}$ and degree exponent of the boxes $d_{k}$. The fractal dimension $d_{B}$ is measured by the scaling of the minimum number of boxes $N_{B}$ of linear size $\ell_{B}$ that is needed to cover the network with order $N$, in other words, $N_{B} / N \sim \ell_{B}^{-d_{B}}$. Similarly,

\footnotetext{
a e-mail: zhangzz@fudan.edu.cn

b e-mail: sgzhou@fudan.edu.cn
}

one can identify the degree exponent of the boxes through the relation $k_{B}\left(\ell_{B}\right) / k_{h u b} \sim \ell_{B}^{-d_{k}}$, where $k_{B}\left(\ell_{B}\right)$ is the degree of each node of the renormalized network, and $k_{h u b}$ the maximum degree of nodes inside each box of original network. In fractal scale-free networks with degree distribution $P(k) \sim k^{-\gamma}$, the three indexes $\gamma, d_{B}$ and $d_{k}$ are related by $\gamma=1+d_{B} / d_{k}[8,9$.

According to the presence of fractal scaling or not, networks can be assorted into two categories [8]: In the presence of fractal behavior, a network is said to be fractal; in contrast, if a network exhibits no fractal scaling, it is defined as non-fractal. Examples of the first class of networks include the World Wide Web (WWW), the actor collaboration networks, metabolic networks, and yeast protein interaction networks. And instances of the second type are the Internet, and most model networks such as the Barabási -Albert (BA) network [7, the Watts-Strogatz (WS) network [6], and the Erdös-Rényi (ER) random graph [10]. In addition to different topological aspects [8, 11, the two types of networks also have distinct consequences regarding the physics of dynamical models running on them [12, 13, 14, 15, 16, 17.

Given the fact that real networks are either fractal or non-fractal, it is consequently of fundamental importance to understand the growth mechanisms and uncover the origins of different kinds of networks. To this end, a wide variety of models have been presented [1,2,4]. However, previous network models, to the best of our knowledge, can generate either fractal networks or non-fractal ones, but rarely both [9]. Thus, it seems quite natural and interesting to set up a unifying framework looking for a deeper 
Zhongzhi Zhang et al.: Transition from fractal to non-fractal scalings in growing scale-free networks

connection between fractal and non-fractal networks. This is the purpose of the current work.

In this paper, by introducing a simple network growth process we propose a unifying scenario for fractal and nonfractal networks. We analytically obtain many structural characteristics of the network, including degree distribution, average path length, diameter, fractal dimension, and betweenness centrality. The degree distribution obeys a power law with an exponent varying continuously. The obtained results on APL and diameter show that the network undergoes a transition from a small- to large-world network. More interestingly, the network exhibits a crossover behavior between fractality and non-fractality.

\section{Network model}

This section is devoted to network construction and computation of some related quantities.

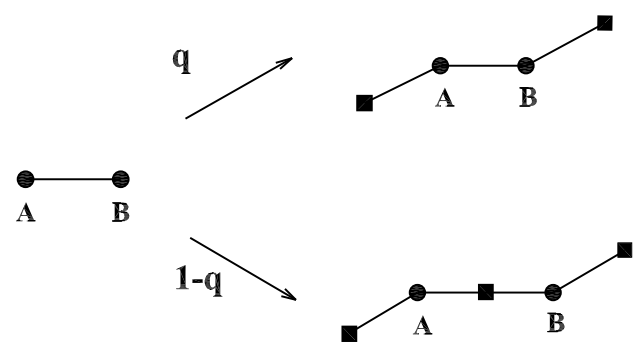

Fig. 1. Iterative construction method of the networks. Each link is replaced by either of the two paths on the right-hand side of the arrow with a certain probability, where each $\mathbf{a}$ stands for a new node.

\subsection{Construction algorithm}

The proposed evolving networks (graphs) have a treelike structure, which are constructed in an iterative way as shown in Fig. 1 Let $T_{t}(t \geq 0)$ denote the networks after $t$ iterations. For $t=0$, the networks growth begins from two nodes (vertices) connected by an edge (link or bond). For $t \geq 1, T_{t}$ is obtained from $T_{t-1}$. We replace each edge in $T_{t-1}$ either by the path on the top right of Fig. 1 with probability $q$, or by the path on bottom right with complementary probability $1-q$. In other words, $T_{t}$ is generated from $T_{t-1}$ by adding $k_{v}$ leaves to each node $v$, where $k_{v}$ is the degree of node $v$ in $T_{t-1}$. Then, the resulting graph is further modified by expanding each edge with probability $1-q$, which was already present in $T_{t-1}$. Expansion of an edge (uv) means: removal of (uv) and creation of an additional node $w$ with edges (uw) and $(v w)$. (Note that real systems may exhibit such an evolving mechanism. For example, this mechanism has been used to model the network evolution of connected minima on a potential energy landscape [18.) This procedure is iterated $t$ times, with the needed graphs obtained in the limit $t \rightarrow \infty$.
As will be shown in next section, when $q=1$, the network is a small world with infinite fractal dimension, its average path length (APL) grows logarithmically with node number. In the particular case of $q=0$, the network is a 'large' world with a finite fractal dimension of 2, its APL scales exponentially with network size. Except these two limiting cases of $q=1$ and $q=0$, for other $q(0<$ $q<1$ ), the networks are growing stochastically. Varying $q$ in the interval $(0,1)$, the networks exhibit a transition from small to large world, and simultaneously show an interesting phenomenon with a transition from non-fractal to fractal behavior.

Note that the current model is similar to the probabilistic model introduced by Song, Havlin, and Makse (SHM Model) in [9. The construction step with probability $q$ in the current manuscript is analogous to the Mode I growth process in the SHM Model, involving strong hubhub attraction. And the step with probability $1-q$ is analogous to the Mode II growth process in the SHM Model, involving hub-hub repulsion. The probabilistic mixing of these two types of construction steps leads to the same results in both of the SHM Model and the current model: when $q=1$ (or pure Mode I) is involved, the network has infinite fractal dimension and shows small-world scaling; any $q \leq 1$ (or mixing of Modes I and II) leads to a finite fractal dimension. However, in [9] only part of the properties was addressed. Here we will present an exhaustive analysis of various characteristics, including additional features (such as average path length and betweenness centrality) not calculated in [9].

\subsection{Order and size}

Now we compute some related quantities such as the number of total nodes and edges in $T_{t}$, called network order and size, respectively. It should be mentioned that as $q$ is a real number, we assume that all variables concerned with $q$ change continuously. Note that a similar assumption was used in Refs. 1, 2, 3, 4, which is valid for large $t$. Let $L_{v}(t)$ be the number of nodes generated at step $t, E_{t}$ the total number of edges present at step $t$. Then $L_{v}(0)=2$ and $E_{0}=1$. By construction (see Fig. 1), at each time step, each existing edge is replaced either by three edges with probability $q$ or by four edges with complementary probability $1-q$. Thus, $E_{t}=q \times 3 E_{t-1}+(1-q) \times 4 E_{t-1}=(4-q)^{t}$ $(t \geq 0)$. At the same time, each existing edge yields two or three new nodes with probability $q$ or $1-q$, this leads to $L_{v}(t)=q \times 2 E_{t-1}+(1-q) \times 3 E_{t-1}=(3-q)(4-q)^{t-1}$ $(t \geq 1)$. Then the number of total nodes $N_{t}$ present at step $t$ is

$$
N_{t}=\sum_{t_{i}=0}^{t} L_{v}\left(t_{i}\right)=(4-q)^{t}+1 .
$$

The average node degree after $t$ iterations is $\langle k\rangle_{t}=\frac{2 E_{t}}{N_{t}}$, which approaches 2 for large $t$, coinciding with the treelike structure of networks. 


\section{Topological properties}

In this section, we will show that the tunable parameter $q$ in the above construction algorithm controls the relevant features of the networks.

\subsection{Degree distribution}

When a new node $i$ is added to the networks at a certain step $t_{i}\left(t_{i} \geq 1\right)$, it has a degree of either 1 or 2 . We denote by $k_{i}(t)$ the degree of node $i$ at time $t$. By construction, the degree $k_{i}(t)$ evolves with time as $k_{i}(t)=2 k_{i}(t-1)$. That is to say, the degree of node $i$ increases by a factor 2 at each time step. Thus, the degree spectrum of the networks is discrete. In network $T_{t}$ all possible degree of nodes is $1,2,2^{2} 2^{3}, \ldots, 2^{t-1}, 2^{t}$.

Let $L_{1}\left(t_{i}\right)$ and $L_{2}\left(t_{i}\right)$ be the separate number of new nodes with degree 1 and 2 that were born at step $t_{i}$. According to the construction algorithm, we have $L_{1}\left(t_{i}\right)=$ $q \times 2 E_{t_{i}-1}+(1-q) \times 2 E_{t_{i}-1}=2(4-q)^{t_{i}-1}$ and $L_{2}\left(t_{i}\right)=$ $(1-q) E_{t_{i}-1}=(1-q)(4-q)^{t_{i}-1}$. Then in network $T_{t}$, the expected number of nodes of degree $k=2^{t-m}$ is $n_{k}=L_{1}(m)+L_{2}(m+1)=\left(6-5 q+q^{2}\right)(4-q)^{m-1}$.

Since the degree spectrum of the networks is not continuous. It follows that the cumulative degree distribution [3] is given by $P_{\text {cum }}(k)=\frac{N_{t, k}}{N_{t}}$, where $N_{t, k}=\sum_{k^{\prime} \geq k} n_{k^{\prime}}$ is the number of nodes whose degree is not less than $k$. When $t$ is large enough, we find $P_{\text {cum }}(k) \approx k^{-\ln (4-q) / \ln 2}$. So the degree distribution $P(k)$ of the networks follows a power-law form $P(k) \sim k^{-\gamma}$ with the exponent

$$
\gamma=1+\frac{\ln (4-q)}{\ln 2}
$$

which is a monotonically decreasing function of $q$. As $q$ increases from 0 to $1, \gamma$ drops form 2 to $1+\frac{\ln 3}{\ln 2}[19$.

\subsection{Average path length}

Shortest paths play an important role both in the transport and communication within a network and in the characterization of the internal structure of the network [5]. Let $d_{i j}$ represent the shortest path length from node $i$ to $j$, then the average path length (APL) $d_{t}$ of $T_{t}$ is defined as the mean of $d_{i j}$ over all couples of nodes in the network, and the maximum value $D_{t}$ of $d_{i j}$ is called the diameter of the network. APL is relevant in many fields regarding real-life networks and has received much attention [20].

For general $q$, it is difficult to derive a closed formula for the APL $d_{t}$ of network $T_{t}$. But for two limiting cases of $q=0$ and $q=1$, both the networks are deterministic ones, which allows one to obtain the analytic solutions for APL. The detailed exact derivation about APL is included in the Appendix section. The obtained results show that the APL for these two particular cases presents qualitatively disparate behaviors: For $q=0$, it is found that

$$
d_{t}=\frac{8+7 \times 4^{t}+13 \times 8^{t}}{14\left(1+4^{t}\right)}
$$

which is approximately equal to $\frac{13 \times 2^{t}}{14}$ for large $t$. Since $N_{t} \sim 4^{t}$ for large network, so $d_{t} \sim N_{t}^{1 / 2}$, indicating that $d_{t}$ grows as a square power of the network order $N_{t}$. This phenomenon is similar to that of the two-dimensional regular lattice [21]. Thus, the network corresponding to $q=0$ is not a small world. For $q=1$, we find

$$
d_{t}=\frac{2\left(1+2 \times 3^{t}+t \times 3^{t}\right)}{3\left(1+3^{t}\right)},
$$

which approximates $\frac{2 t}{3}$ in the infinite $t$, implying that the APL shows a logarithmic scaling with network order. Therefore, in the specific case of $q=1$, the network exhibits a small-world behavior.

Thus, when we tune $q$ from 0 to 1 , the networks undergo a transition from a 'large' to small world. We stress that such a transition has already been observed in some previously studied models 22 .

\subsection{Diameter}

As mentioned in preceding subsection, the diameter of a network is defined as the longest shortest path between all pairs of nodes, characterizing the maximum communication delay in the network. Although we do not give a closed formula of APL of $T_{t}$ for general $q$, here we will provide the exact result of the diameter of $T_{t}$ denoted by $D_{t}$.

We first address the network of $q=1$ case, where the shortest distances between existing node pairs are not altered when new nodes enter the systems. For this particular case, $D_{0}=1$. At each time step, the diameter of the network increases by 2 . Then the diameter of $T_{t}$ is $D_{t}=2 t+1$ and thus scales logarithmically with the network order, showing a similar behavior as that of the average path length. Since small diameter is consistent with the concept of 'small-world', the additive growth in the diameter with time also (as the APL) suggests that the network for $q=1$ case is a small world.

For $0 \leq q<1$, the addition of new nodes affects fundamentally the distances between existing node pairs. By construction algorithm, for any existing couple of nodes connected by a link, after a generation of evolution, the distant $l$ between this pair of nodes may be equal to 1 or 2 with probability $q$ and $1-q$, respectively. Thus the expected value of $l$ is $2-q$. Using this result and considering that the networks are treelike, we can derive the following recursive relation for expected $D_{t}$ :

$$
D_{t}=(2-q) D_{t-1}+2 \text {. }
$$

Since $D_{0}=1$, we can resolve Eq. (5) to obtain the average of network diameter as

$$
D_{t}=\left(1+\frac{2}{1-q}\right)(2-q)^{t}-\frac{2}{1-q},
$$

which grows as a power of time $t$. 


\subsection{Fractal dimension}

To determine the fractal dimension, we distinguish two cases: $0 \leq q<1$ and $q=1$. In the case of $0 \leq q<$ 1 , we follow the mathematical framework introduced in Ref. 9]. We are concerned about three quantities: network order $N_{t}$, network diameter $D_{t}$, and degree $k_{u}(t)$ of a given node $u$. By construction, we can easily see that in the infinite $t$ limit, these quantities grow obeying the following relations: $N_{t} \simeq(4-q) N_{t-1}, D_{t} \simeq(2-q) D_{t-1}, k_{u}(t)=$ $2 k_{u}(t-1)$. Thus, for large networks, $N_{t}, k_{u}(t)$ and $D_{t}$ increase by a factor of $f_{N}=4-q, f_{k}=2$, and $f_{D}=2-q$, respectively.

From above obtained microscopic parameters demonstrating the mechanism for network growth, we can derive the scaling exponents: the fractal dimension $d_{B}=\frac{\ln f_{N}}{\ln f_{D}}=$ $\frac{\ln (4-q)}{\ln (2-q)}$ and the degree exponent of boxes $d_{k}=\frac{\ln f_{k}}{\ln f_{D}}=$ $\frac{\ln 2}{\ln (2-q)}$. According to the scaling relation of fractal scalefree networks, the exponent of the degree distribution satisfies $\gamma=1+\frac{d_{B}}{d_{k}}=1+\frac{\ln (4-q)}{\ln 2}$, giving the same $\gamma$ as that obtained in the direct calculation of the degree distribution, see Eq. (2).

For $q=1$, although the number of their nodes increases exponentially, its diameter grows linearly with time. Thus, in this case the network has infinite dimension and does not present a fractal topology.

\subsection{Betweenness centrality}

Betweenness centrality (BC) of a given node is the accumulated fraction of the total number of shortest paths going through the node over all node pairs [24,23]. More precisely, the betweenness of a node $i$ is

$$
b_{i}=\sum_{j \neq i \neq k} \frac{\sigma_{j k}(i)}{\sigma_{j k}}
$$

where $\sigma_{j k}$ is the total number of shortest path between node $j$ and $k$, and $\sigma_{j k}(i)$ is the number of shortest path running through node $i$.

We now investigate the $\mathrm{BC}$ distribution of nodes. It was claimed in previous studies that for scale-free networks, the BC distribution $P(b)$ of nodes obeys a power law with the exponent $\gamma_{b}=2$, i.e., $P(b) \sim b^{-2}$. It was also suggested that the exponent $\gamma_{b}=2$ is universal for all scale-free networks [25]. Next, we will present that the exponent $\gamma_{b}$ is not universal and constant (at least for fractal trees), but varies significantly as a function of $d_{B}$ (or a function or $q$ ).

In order to obtain the exponent $\gamma_{b}$ of $\mathrm{BC}$ distribution of our networks, we resort to a heuristic argument similar to that applied in 26. Notice that all of our networks are treelike and fractal (except the case of $q=1$ ). Considering one network with dimension $d_{B}$, for a small area of the network containing $g$ nodes, its average path length is typically $d(g)=g^{1 / d_{B}}$ [27]. All nodes in this small region can reach the rest nodes in the network via $d(g)$ nodes.
Thus, the BC of these $d(g)$ nodes is not less than $g$. On the other hand, in the whole network there are $N_{t} / g$ such areas, each of which includes $g$ nodes. Then, the total number of nodes with BC $b$ not less than $g$ in the whole network is

$$
n(b \geq g) \sim d(g) \times \frac{N_{t}}{g} \sim N_{t} \times g^{-\left(1-1 / d_{B}\right)} .
$$

Therefore, the cumulative $\mathrm{BC}$ distribution is

$$
P_{c u m}(b)=\frac{n(b \geq g)}{N_{t}} \sim g^{-\left(1-1 / d_{B}\right)},
$$

which implies $P(b) \sim b^{-\gamma_{b}}$ with

$$
\gamma_{b}=2-\frac{1}{d_{B}} .
$$

Thus, $\gamma_{b}$ is a increasing function of fractal dimension $d_{B}$, the larger the fractal dimension $d_{B}$, the larger the exponent $\gamma_{b}$. Eq. (10) shows that $\gamma_{b}=2$ holds only for the non-fractal networks with $d_{B} \rightarrow \infty$ [28, where a relatively small number of hub nodes bear large BC. In contrast, in fractal networks, the BC of a lot of 'small' nodes can be compared with that of hub nodes.

\section{Conclusions}

In the paper, by introducing a parameter $q$, we have presented a simple network growth process to generate a unified model for fractal and non-fractal networks. This process was shown to lead to a rich behavior for the network structure. Various relevant topological properties have been determined depending on the model parameter $q$. It has been shown that both degree and betweenness centrality distributions of nodes have a power-law tail and the characteristic exponents change continuously with the parameter $q$. Other structural properties including the APL, diameter, and fractal dimension have been obtained as well, which indicate that the model undergoes a crossover from large-world to small-world networks, and simultaneously exhibits a transition from fractal to non-fractal behaviors.

In spite of the simplicity, our minimal model can capture the essential characteristics and correlations of fractal and non-fractal networks. It is helpful for understanding the growth mechanisms and evolutions of the two different sorts of networks. Finally, it should be mentioned that, although we only studied treelike networks, in a similar way, one can construct models considering the effect of loops, whose general properties are similar to those of the model investigated in the present work.

\section{Acknowledgment}

We thank Yichao Zhang for preparing this manuscript. This research was supported by the National Basic Research Program of China under grant No. 2007CB310806, the National Natural Science Foundation of China under 
Grant Nos. 60496327, 60573183, 60773123, and 60704044, the Shanghai Natural Science Foundation under Grant No. 06ZR14013, the Postdoctoral Science Foundation of China under Grant No. 20060400162, Shanghai Leading Academic Discipline Project No. B114, the Program for New Century Excellent Talents in University of China (NCET-06-0376), and the Huawei Foundation of Science and Technology (YJCB2007031IN).

\section{Appendix A: Derivation of the average path length for two limited cases}

Following an algebraic method similar to that introduced in [12, we can compute the average path length (APL) for two limiting deterministic cases. By definition, the APL for $T_{t}$ is defined as

$$
d_{t}=\frac{S_{t}}{N_{t}\left(N_{t}-1\right) / 2},
$$

where

$$
S_{t}=\sum_{i \in T_{t}, j \in T_{t}, i \neq j} d_{i j}
$$

denotes the sum of the shortest path length between two nodes over all pairs. For the two particular cases $q=0$ and $q=1$, Both of the networks have a self-similar structure that allows one to calculate $d_{t}$ analytically. The selfsimilar structure is obvious from an equivalent network construction method: to obtain $T_{t+1}$, one can make some copies of $T_{t}$ and join them in the hub nodes.

\section{The $q=0$ case}

As shown in Fig. 2, for the $q=0$ case, the network $T_{t+1}$ may be obtained by the juxtaposition of four copies of $T_{t}$, which are labeled as $T_{t}^{\theta}, \theta=1,2,3,4$. Then we can write the sum $S_{t+1}$ as

$$
S_{t+1}=4 S_{t}+\Delta_{t}
$$

where $\Delta_{t}$ is the sum over all shortest paths whose endpoints are not in the same $F_{t}$ branch. The solution of Eq. A.3 is

$$
S_{t}=4^{t-1} S_{1}+\sum_{x=1}^{t-1} 4^{t-x-1} \Delta_{x}
$$

The paths that contribute to $\Delta_{t}$ must all go through at least one of the three edge nodes (i.e., $\boldsymbol{E}, \boldsymbol{F}$ and $\boldsymbol{G}$ in Fig. 2(b)) at which the different $T_{t}$ branches are connected. The analytical expression for $\Delta_{t}$, called the crossing paths, is found below.

Denote $\Delta_{t}^{\alpha, \beta}$ as the sum of all shortest paths with endpoints in $T_{t}^{\alpha}$ and $T_{t}^{\beta}$. If $T_{t}^{\alpha}$ and $T_{t}^{\beta}$ meet at an edge node, $\Delta_{t}^{\alpha, \beta}$ rules out the paths where either endpoint is that shared edge node. If $T_{t}^{\alpha}$ and $T_{t}^{\beta}$ do not meet, $\Delta_{t}^{\alpha, \beta}$ excludes the paths where either endpoint is any edge node. Then the total sum $\Delta_{t}$ is

$$
\Delta_{t}=\Delta_{t}^{1,2}+\Delta_{t}^{1,3}+\Delta_{t}^{1,4}+\Delta_{t}^{2,3}+\Delta_{t}^{2,4}+\Delta_{n}^{3,4} .
$$

By symmetry, $\Delta_{n}^{1,2}=\Delta_{t}^{2,3}=\Delta_{n}^{3,4}$ and $\Delta_{t}^{1,3}=\Delta_{t}^{2,4}$, so that

$$
\Delta_{t}=3 \Delta_{t}^{1,2}+2 \Delta_{t}^{1,3}+\Delta_{t}^{1,4} .
$$

In order to find $\Delta_{t}^{1,2}, \Delta_{t}^{1,3}$, and $\Delta_{t}^{1,4}$, we define

$$
s_{t}=\sum_{i \in T_{t}^{1}, i \neq E} d_{i, E} .
$$

Considering the self-similar network structure, we can easily know that at time $t+1$, the quantity $s_{t+1}$ evolves recursively as

$$
\begin{aligned}
s_{t+1} & =2 s_{t}+\left[s_{t}+2^{t}\left(N_{t}-1\right)\right]+\left[s_{t}+2^{t+1}\left(N_{t}-1\right)\right] \\
& =4 s_{t}+3 \times 2^{3 t} .
\end{aligned}
$$

Using $s_{1}=7$, we have

$$
s_{t}=2^{2 t-2}+3 \times 2^{3 t-2} .
$$

On the other hand, by definition given above, we have

$$
\begin{aligned}
\Delta_{t}^{1,2} & =\sum_{\substack{i \in T_{t}^{1}, j \in T_{t}^{2} \\
i, j \neq E}} d_{i j} \\
& =\sum_{\substack{i \in T_{t}^{1}, j \in T_{t}^{2} \\
i, j \neq E}}\left(d_{i E}+d_{j E}\right) \\
& =\left(N_{t}-1\right) \sum_{\substack{i \in T_{t}^{1} \\
i \neq E}} d_{i E}+\left(N_{t}-1\right) \sum_{j \in T_{t}^{2}} d_{j E} \\
& =2\left(N_{t}-1\right) \sum_{\substack{i \in T_{t}^{1} \\
j \neq E}} d_{i E} \\
& =2\left(N_{t}-1\right) s_{t}, \Delta_{t}^{1,3}=\sum_{\substack{i \in T_{t}^{1}, i \neq E \\
j \in T_{t}^{3}, j \neq F}} d_{i j} \\
& =\sum_{\substack{i \in T_{t}^{1}, i \neq E \\
j \in T_{t}^{3}, j \neq F}}^{2\left(N_{t}-1\right) s_{t}+\left(N_{t}-1\right)^{2} \times 2^{t},} \\
& \left.=d_{i E}+d_{E F}+d_{j F}\right)
\end{aligned}
$$

and

$$
\begin{aligned}
\Delta_{t}^{1,4}= & \sum_{\substack{i \in T_{t}^{1}, i \neq E \\
j \in T_{t}^{4}, j \neq G}} d_{i j} \\
= & \sum_{\substack{i \in T_{t}^{1}, i \neq E \\
j \in T_{t}^{4}, j \neq G}}\left(d_{i E}+d_{E G}+d_{j G}\right) \\
= & 2\left(N_{t}-1\right) s_{t}+\left(N_{t}-1\right)^{2} \times 2^{t+1},
\end{aligned}
$$




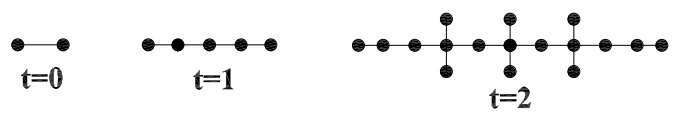

(a)

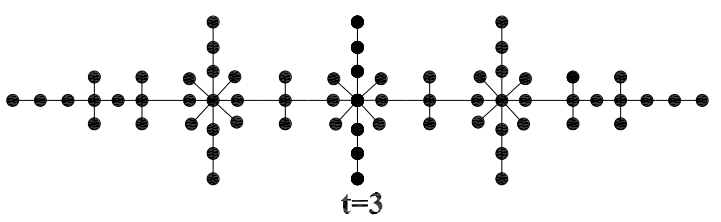

(b)

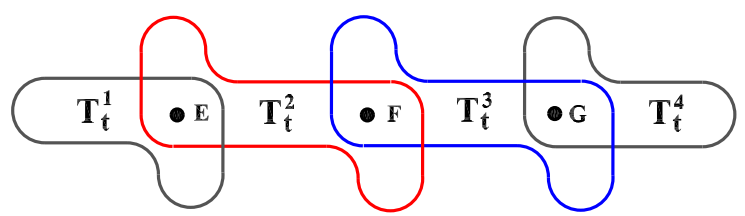

Fig. 2. (Color online) The network growth process for the particular case $q=0$. (a) Illustration of the first four evolution steps. (b) Second construction method of the network for $q=0$ case that highlights self-similarity: The graph after $t+1$ construction steps, $T_{t+1}$, is composed of four copies of $T_{t}$ denoted as $T_{t}^{\theta}(\theta=1,2,3,4)$, which are connected to one another as above.

where $d_{E F}=2^{t}$ and $d_{E G}=2^{t+1}$ have been used. Substituting Eqs. (A.10), A.11 and (A.12) into Eq. A.6 , we obtain

$$
\begin{aligned}
\Delta_{t} & =12\left(N_{t}-1\right) s_{t}+4\left(N_{t}-1\right)^{2} \times 2^{t} \\
& =13 \times 32^{t}+3 \times 16^{t} .
\end{aligned}
$$

Inserting Eqs. A.13 for $\Delta_{x}$ into Eq. A.4, and using $S_{1}=20$, we have

$$
S_{t}=\frac{4^{t}}{28} \times\left(8+7 \times 4^{t}+13 \times 8^{t}\right) .
$$

Inserting Eq. A.14 into Eq. A.1 , one can obtain the analytical expression for $d_{t}$ in Eq. (3).

\section{The $q=1$ case}

Using analogous analysis for the $q=0$ case, we can calculate the APL $d_{t}$ for the case of $q=1$. For simplicity, we use the same symbols used in last subsection to represent the identical notions. For $q=1$ case, the network $T_{t+1}$ may be obtained by joining at the hubs (the most connected nodes) three copies of $T_{t}$ labeled as $T_{t}^{(\psi)}, \psi=1,2,3$ [29], see Fig. 3. Then one can write the sum over all shortest paths $S_{t+1}$ as

$$
S_{t+1}=3 S_{t}+\Delta_{t} .
$$

The solution of Eq. A.15 is

$$
S_{t}=3^{t-1} S_{1}+\sum_{\tau=1}^{t-1} 3^{t-\tau-1} \Delta_{\tau} .
$$

The paths contributing to $\Delta_{t}$ must all go through at least either of the two hubs $(X$ and $Y$ ) where the three different $T_{t}$ branches are joined. The crossing paths $\Delta_{t}$ is given by

$$
\Delta_{t}=\Delta_{t}^{1,2}+\Delta_{t}^{2,3}+\Delta_{t}^{1,3}
$$

where $\Delta_{t}^{1,2}$ rules out the paths where either endpoint is node $X, \Delta_{t}^{2,3}$ rules out the paths where either endpoint is node $Y$, and $\Delta_{t}^{1,3}$ excludes the paths with an endpoint is either $X$ or $Y$. Again by symmetry, $\Delta_{t}^{1,2}=\Delta_{t}^{2,3}$, so that

$$
\Delta_{t}=2 \Delta_{t}^{1,2}+\Delta_{t}^{1,3} \text {. }
$$

In this case, the quantity $s_{t+1}$ evolves as

$$
\begin{aligned}
s_{t+1} & =2 s_{t}+\left[s_{t}+\left(N_{t}-1\right)\right] \\
& =3 s_{t}+3^{t} .
\end{aligned}
$$

Since $s_{1}=4$, Eq. A.19 is solved inductively:

$$
s_{t}=(t+3) \times 3^{t-1} \text {. }
$$

similarly,

$$
\begin{aligned}
\Delta_{t}^{1,2} & =\sum_{\substack{i \in T_{t}^{1}, j \in T_{t}^{2} \\
i, j \neq X}}\left(d_{i X}+d_{j X}\right) \\
& =2\left(N_{t}-1\right) s_{t}
\end{aligned}
$$

and

$$
\begin{aligned}
\Delta_{t}^{1,3} & =\sum_{\substack{i \in T_{t}^{1}, i \neq X \\
j \in T_{t}^{3}, j \neq Y}}\left(d_{i X}+d_{X Y}+d_{j Y}\right) \\
& =2\left(N_{t}-1\right) s_{t}+\left(N_{t}-1\right)^{2} .
\end{aligned}
$$

Substituting the obtained expressions in Eqs. A.21 and (A.22) into Eq. (A.18), the crossing paths $\Delta_{t}$ is found to be

$$
\Delta_{t}=7 \times 9^{t}+2 t \times 9^{t} .
$$

Inserting Eq. A.23 into Eq. A.16 and using the initial condition $S_{1}=10$, we have

$$
S_{t}=3^{-1+t}\left(1+2 \times 3^{t}+t \times 3^{t}\right) .
$$

Substituting Eq. A.24 into (A.1), the exact expression for the average path length is obtained as shown in Eq. (4). 
(a)
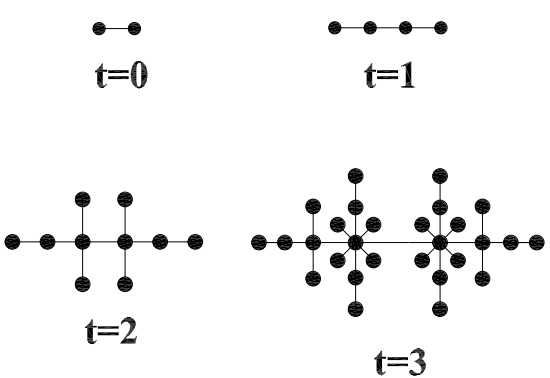

(b)

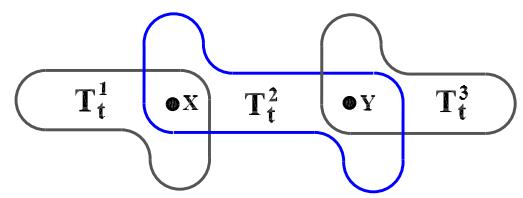

Fig. 3. (Color online) The network growth process for the specific case $q=1$. (a) Scheme of the first four iterative processes. (b) Second construction means of the network for $q=1$ case: The graph after $t+1$ construction steps, $T_{t+1}$, can be obtained by joining three copies of $T_{t}$ denoted as $T_{t}^{\psi}(\psi=1,2,3)$, which are connected to each other at the two edge nodes $(X$ and $Y)$.

\section{References}

1. R. Albert and A.-L. Barabási, Rev. Mod. Phys. 74, 47 (2002).

2. S.N. Dorogovtsev and J.F.F. Mendes, Adv. Phys. 51, 1079 (2002).

3. M.E.J. Newman, SIAM Rev. 45, 167 (2003).

4. S. Boccaletti, V. Latora, Y. Moreno, M. Chavez and D.-U. Hwanga, Phys. Rep. 424, 175 (2006).

5. L. da. F. Costa, F.A. Rodrigues, G. Travieso, and P.R.V. Boas, Adv. Phys. 56, 167 (2007).

6. D.J. Watts and H. Strogatz, Nature (London) 393, 440 (1998).

7. A.-L. Barabási and R. Albert, Science 286, 509 (1999).

8. C. Song, S. Havlin, H. A. Makse, Nature 433, 392 (2005).

9. C. Song, S. Havlin, H. A. Makse, Nature Phys. 2, 275 (2006).

10. P. Erdös, A. Rényi, Pub. Math. Insti. Hung. Acad. Sci. 5 (1960) 17.

11. M. Kitsak, S. Havlin, G. Paul, M. Riccaboni, F. Pammolli, and H. E. Stanley, Phys. Rev. E 75, 056115 (2007).

12. M. Hinczewski and A. N. Berker, Phys. Rev. E 73, 066126 (2006).

13. Z. Z. Zhang, S. G. Zhou, and T. Zou, Eur. Phys. J. B 56, 259 (2007).

14. Z. Z. Zhang, S. G. Zhou, T. Zou, and J. H. Guan, (unpublished).

15. H. D. Rozenfeld, S. Havlin, and D. ben-Avraham, New J. Phys. 9, 175 (2007).

16. H. D. Rozenfeld and D. ben-Avraham, Phys. Rev. E 75, 061102 (2007).

17. M. Hinczewski, Phys. Rev. E 75, 061104 (2007).

18. C. P. Massen and J. P. K. Doye, J. Chem. Phys. 127, 114306 (2007).

19. S. Jung, S. Kim, and B. Kahng, Phys. Rev. E 65, 056101 (2002).

20. A. Fronczak, P. Fronczak, and J. A. Hołyst, Phys. Rev. E 70, 056110 (2004); J. A. Hołyst, J. Sienkiewicz, A. Fronczak, P. Fronczak, and K. Suchecki, Phys. Rev. E 72,
026108 (2005); S. N. Dorogovtsev, J. F. F. Mendes, and J. G. Oliveira, Phys. Rev. E 73, 056122 (2006); Z.Z. Zhang, L.C. Chen, S.G. Zhou, L.J. Fang, J.H. Guan, and T. Zou, Phys. Rev. E 77, 017102 (2008).

21. M. E. J. Newman, J. Stat. Phys. 101819 (2000).

22. K. Klemm and V. M. Eguíluz, Phys. Rev. E 65, 057102 (2002); L. Tian, C.-P. Zhu, D.-N. Shi, Z.-M. Gu, and T. Zhou, Phys. Rev. E 74, 046103 (2006); Z. Z. Zhang, S. G. Zhou, Z. Shen, and J. H. Guan, Physica A 385, 765 (2007); Z. Z. Zhang, S. G. Zhou, Z. Y. Wang, and Z. Shen, J. Phys. A 40, 18863 (2007); S. N. Dorogovtsev, P. L. Krapivsky, and J. F. F. Mendes, Europhys. Lett. 81, 30004 (2008).

23. M. E. J. Newman, Phys. Rev. E 64, 016132 (2001).

24. C. L. Freeman, Sociometry 40, 35 (1977).

25. K.-I. Goh, B. Kahng, and D. Kim, Phys. Rev. Lett. 87, 278701 (2001).

26. L. A. Braunstein, Z. Wu, Y. Chen, S. V. Buldyrev, T. Kalisky, S. Sreenivasan, R. Cohen, E. López, S. Havlin, and H. E. Stanley, Int. J. Bifurcation Chaos 17, 2215 (2007).

27. Fractals in Science, edited by A. Bunde and S. Havlin (Springer, Berlin, 1996).

28. C.-M. Ghima, E. Oh, K.-I. Goh, B. Kahng, and D. Kim, Eur. Phys. J. B 38, 193 (2004); Z. Z. Zhang, S. G. Zhou, L. C. Chen, J. H. Guan, L. J. Fang, and Y. C. Zhang, Eur. Phys. J. B 59, 99 (2007).

29. E. Bollt, D. ben-Avraham, New J. Phys. 7, 26 (2005); F. Comellas, H. D. Rozenfeld, D. ben-Avraham, Phys. Rev. E 72, 046142 (2005). 